tists then had painful ways; he sat upon a bench, and took your head between his knees, and, muttering, "Look pleasant, please," he plied his monkey wrench. It took six men to hold me down when he adjusted bridge or crown, or plugged a hollow fang, and travelers could hear me roar away upon the distant shore of Yang-tsze-kiang. But now I like the dentist's chair, I like to sit and rest me there, from morning until dusk, to sit in comfort and to snooze, and have the gentle dentist use his forceps on my tusk. WALT MASON."

1. Maudsley, Physiology of Mind, $p$. 302.

2. Carpenter, Physiology of Mind, $p$. 302.

3. Hack Tuke, Mind and Body, vol. 1, p. 2.

4. Ibid, p. 16.

5. Schofield, The Unconscious Mind, p. 257.
6. W. James, Psychology, vol. ii, p. 602,

7. Braid, Power of Mind Over Body, p. 6 .

8. Schofield, The Unconscious Mind, p. 259 .

9. W. T. Reeves, The Use of Comprest Air for Desensitizing Sensitive Dentine, Dental Review, Sept. 1913, p. 905 .

10. DeFord, Comfortable and Dependable Dental Operations, Dental Summary, Nov. 1913 , p. 845.

11. DeFord, Analgesia in Dentistry, Dental Review, Sept., 1913, p. 896.

12. Hewitt, Anesthetics, Third Edition, p. 84 .

13. DeFord, Lectures on General Anesthetics in Dentistry, Second Edition, p. 267.

14. DeFord, Comfortable and Dependable Dental Operations, Dental Summary, Nov. 1913 , p. 842.

15. Crile, Shock, Journal of American Medical Association, Dec. 6, 1913, p. 2027.

\title{
NITROUS OXID AND OXYGEN ANALGESIA IN OPERATIVE DENTISTRY.
}

\author{
By J, P. Henahan, D. D. S., Cleveland, Ohio.
}

$\mathrm{I}$ AM deeply mindful and appreciative of the honor which permits of my reading a paper to this famous body. I regret only the personal limitations which prevent a greater and more worthy effort.

Dentistry during the past years has developed and benefited by such a variety of improvements in ideas and methods of practise, that if a Rip Van Winkle were to awaken today and behold our profession, he would scarcely recognize in it the comparatively crude art of twenty years ago.

Not least among the improvements, is the realization of that for which the dental procession has labored so long and persistently, namely, the successful application of anesthesia in operative dentistry. Several methods have been developed, each possessing its peculiar virtues, and all long since past the experimental stage.

With the rapid advances which are be- 
ing made in all other branches of our work, it is only reasonable to expect that this will keep stride, and in a few years, such a revolution will have taken place in dental practises, that some extraordinary condition alone will be excuse for a dentist inflicting pain upon a patient. His education and training will enable him to make a scientific selection of the best method for any given case, not relying upon any one method to suffice under all conditions.

The part has been allotted to me to present for consideration by this convention, the virtues of the agent which, in my opinion, holds the greatest possibilities for the greater number, laity as well as profession, considered. The agent which will reward the effort made to master its peculiarities, with the most nearly universal success, and the one which long usage has shown to be attended by no peculiarities which manifest themselves in dangerous after effects.

After reviewing statistics and clinical data, and also the results of several years actual experience in which Nitrous Oxid and Nitrous Oxide and Oxygen have been employed by me as an anesthetic and analgesic in general surgery, as well as in every variety of dental cases, I feel no hesitancy in recommending this agent to the members of the dental profession as a remedy for prevention wherever pain may be caused thru the performance of dental operations.

It is not my purpose to burden you with the presentation of a scientific treatise upon Nitrous Oxide and Oxygen, its chemical character or its physiological action, nor to present any new or socalled original method for its administration, but simply to remind my hearers of the possibilities, in the line of humanitarian dentistry, to be gained thru the medium of its employment.

Neither should it be necessary to make any comparison of Nitrous Oxid and Nitrous Oxid and Oxygen with the other well known anesthetic agents so commonly employed today. Hundreds of articles have been written, which deal with theories of the probable manner thru which anesthesia is produced, and also statistical records showing effects which manifest themselves days after the patient has apparently recovered from the anesthetic effects of the particular drug. In all these comparisons Nitrous Oxid and Oxygen has been conceded the position of being the most desirable general anesthetic in use.

These opinions all tend to show that when the dentist employs Nitrous Oxid and Oyxgen in a sensible manner, he is employing the anesthetic in which is embodied the least possible danger.

Altho anesthesia is a subject of the greatest importance to the dentist, and in itself necessary to him as the only method which makes possible the prevention of pain in many of his operations, still, as it is not the intention of this symposium to consider anesthesia but analgesia, we will pass at once to our subject.

Analgesia may be defined as that state in which the appreciation of pain is lost or materially lowered without the loss of the sense of touch or consciousness.

For the practise of analgesia a general plan of procedure should be adopted which, with necessary variations for individual cases, should be carried out with every patient.

The more simple the plan the greater its virtue. Under the plan the patient's mind should be put at perfect ease and no intimation of any burden of responsibility should be allowed to exist.

By this is meant that the patient should not be led to believe that upon him rests the responsibility of making known to the dentist the symptoms which characterize the development of analgesia. I have reason to believe that instructions along this line are often the cause of failure, due to bewilderment and doubt created in the minds of some, 
which caused them to withhold confessions of conditions until too late, and the exciting stage of anesthesia has been reached.

I have outlined the following plan which, for purposes of explanation, may be divided into four parts.

1st. Preliminary instruction.

2nd. Suggestion.

3rd. Application of anesthetic.

4th. Performance of operation.

In practise each of these four divisions should be given its due consideration, if uniform success is to crown our efforts. Disregard will be rewarded usually with disappointment.

1st. The first part consists of information and preparation regarding diet. Usually aim to have the same condition as for anesthesia, a fasting condition for three hours, and in some cases nothing short of the most complete preparation regarding fast will permit of the continuation of analgesia for any length of time. Nausea and vomiting will result, and together with the resultant depression will terminate our efforts for the time being at least.

\section{2nd suggestion.}

Under this heading we must consider our entire behavior toward the patient. In some exaggerated cases our every word and action must be carefully calculated to create the proper trend of ideas in the patient's mind.

Originality and discernment will be necessary to enable one to deal successfully with the various types which he will meet.

The bearing of the operator is of the first importance. He should be dignified and composed himself, if he hopes to instill the patient with confidence.

All our plans, including the explanation of the action and effect of the anesthetic, really are for the purpose of suggestion, to inspire the patient with confidence that no pain is to be experienced.

Many patients, especially those who have had some experience with analge- sia, will not require much more attention in this regard than the simple statement of the purpose for which the measure is to be resorted to and the results to be attained.

The explanation of the purpose and method of administration should be brief but concise. It should in all cases, state that the condition of unconsciousness is not desired, that we are depending upon patient for some slight assistance which requires him being in a conscious condition.

Make plain that while there shall be no lack of consciousness, no pain what ever shall be experienced.

In cases where necessary, state that co-operation on patient's part is essential to success.

Neither after beginning of analgesia, nor at any other stage should any action or expression be allowed which might convey an impression that the operator, himself, lacks confidence. Never ask if it hurts. This question exposes you to patient as not having complete control of everything and impairs their confidence.

Visitors with patients should not be permitted to talk to or hold hands, or in any other manner interfere with the patient.

No noise or loud talking should under any circumstance be permitted in the operating room. The more tranquil and quiet a patient is allowed to be the greater the chance for success.

3rd. Application of anesthetic.

Under the heading we must first consider our machine and gas supply.

In this matter I believe that one may enjoy good results, altho employing the simplest or most primitive of machines, providing his experience has enabled him to develop technic, but as in other classes of work, I believe that good and modern instruments tend to improve our results. In the case of beginners a good machine is indispensable.

The principle virtue of any machine is 
simplicity. It should be such that the gas is controlled in such a manner that we may increase or decrease our supply instantly and at all times know exactly the quantity we are permitting our patients to inhale.

The inhaler also should be of simple construction, permitting the greatest range of admixture of gases with the atmosphere.

Before adjusting the inhaler to nose see that air ways are completely open; also all necessary valves on machine except the one for releasing gas. Allow Nitrous Oxid bag to be almost collapsed.

Now instruct patient that all that will be necessary will be to continue in the ordinary manner without any special effort, breathing entirely thru the nose.

Apply inhaler and discourage all attempts of patient to talk. Release a small amount of gas, say at rate of 20 gallons per hour; after this allow four minutes to pass without any interference at all with patient, at which time, in the average case the patient will be in a condition to allow of a very careful beginning of operation.

At this point we are allowing patient to breath a mixture which contains a maximum of atmosphere as a diluent, and we are not entirely certain that our patient is in the proper condition for the operation. We must ascertain in the only available manner open to us, by beginning to operate.

4th. Performance of operation.

This is the critical moment, we should exercise the greatest caution about inflicting any pain.

We must not forget that the patient's mind is full of doubt and we must dispel this doubt and replace it with confidence. Therefore all depends upon the manner in which we begin our operation.

It is advisable also that in planning our operation, we should arrange for a continuation of our work, one preparation after another without any unnecessary interruption or delay as some patients will become more or less excited upon taking up work after each interruption.

During the early stages especially, many patients will flinch and pull away, but we must be sure that this is not due to fear rather than pain, before changing the anesthetic mixture. If, however, we find that it is necessary to produce a deeper condition, we may do so either by excluding some atmosphere, thereby enriching our mixture in that way, or by releasing more gas.

In this we must always bear in mind that the longer the duration of the analgesia, the deeper the condition has a tendency to become with a given amount of gas. We must therefore be watchful, for if the patient crosses the margin into the exciting stage, it often becomes difficult to operate, due to unrest of patient.

Analgesia may be deepened and lightened to meet the requirements of the operation by manipulation of air valves on inhaler.

If, as we see sometimes in the case of anemic young men and women some cyanosis is evident, despite the presence of so much air, the condition may be cleared up by the addition of a small amount of oxygen to the mixture.

As in anesthesia, we will meet with patients who will cause trouble and an. noyance during any attempt to produce analgesia in their case.

Here again experience will tell inasmuch as it enables one to often recognize certain types and thereby save oneself disagreeable experience by not attempting at all to analgesize patient or to quickly recognize the futility of persistence after a reasonable attempt has been made.

The patient who may thus interfere with the success of our efforts may be classified after a more or less general plan.

The patient most sure to bring failure is the one who is accustomed to the use of strong drink as matter of habit, but the evidence of a recent indulgence, in 
the case of any patient, should be sufficient reason to postpone the attempt to induce analgesia; the effect of a few inhalations of Nitrous Oxid and Oxygen upon the patient who has imbibed only a single glass of whiskey, appears to be able to stir to action about all the devils not confined in Hades.

Habitual drug users, either as an uncontrollable habit or as a medical treatment, will be found to be unreliable. Those habitual narcotic users will, when in need of the fortifying effects of a dose of the drug, befound impossible to control. If the condition is suspected, not much time should be wasted in their case.

Patients who make no effort to co-operate with dentist, due to inability, or refusal to exert will power, will be found to be about as unsatisfactory a case as can be imagined; suggestion in these cases avails us nothing for the reason that patient will not dwell upon our statements.

Hysterical patients may, thru care. ful handling, sometimes be successfully treated thru the power of suggestion plus analgesia, but in every instance caution is advised.

This class of patients produced our most interesting as well as sometimes alarming results; a single inhalation may be sufficient to enable us to complete extensive operations, or we may see conditions develop which may be due to hysteria.

Patients sometimes present, who, under ordinary conditions, may be regarded as average patients in every respect, but who, as the result of some severe mental or physical strain, may temporarily be so hysterical under analgesia as to prevent, or at least, limit our success.

Nausea or vomiting are causes of failure which we sometimes have to contend with.

I have found that in these cases our trouble may, as a rule be ascribed to one of two reasons. Either we have failed to prepare patient properly in regard to fasting, or the patient is a sufferer from some form of stomach trouble and will present a history of easily provoked nausea.

There is an occasional patient, usually a man, in whom there seems to be no margin between consciousness and the exciting stage of anesthesia, in which, due to the unrest of the patient, it is often found impossible to accomplish much in the line of operating.

Children, when amenable to reason, are usually very satisfactory patients for analgesia, a small amount of anesthetic sufficing to produce excellent results. The child, who cannot be prevailed upon to submit quietly, can seldom be treated with full success, but anything at all accomplished is that much gained.

After having acquired more or less experience along this line, the dentist will have developed some technic. From this time on his efforts will be rewarded by more satisfactory and uniform results. I believe that on an average $98 \%$ of our attempts will be successful under proper conditions.

Under perfect analgesia nearly all operations which come under the head of operative dentistry may by performed painlessly.

Removal of nerves is not advised, but after exposure has been made, cocaine may be applied, patient still under analgesia, and nerve removed in this manner.

Severe extractions also are not advised, altho simple extractions are possible.

All other proceedings may be brought to a satisfactory termination under this condition.

It should hardly be necessary to mention the advantages which may accrue as the result of anesthesia in operative dentistry.

The advantages are many and result equally to dentist and patient.

The percentage of people who procras- 
tinate in having dental work performed, due to fear of pain, must be very large. We owe it to the public as a professional duty, each one of us, to exert our best efforts to become proficient in any method which will enable those people to avail themselves of our services.

On our own part, we will enjoy the advantage of increased efficiency, more work accomplished and no work only partly done, due to insufficient preparation on account of pain.

We will benefit by the conservation of nervous energy which would be wasted upon patient who is unable to withstand pain of operation.
The day of painless dentistry has dawned and every dentist may as well realize that unless he equips himself to meet the issue with the rest, he will surely lose ground. We must realize that there is no middle position - to achieve success, we cannot stand still, we must move forward or we are falling behind as the rest of the world moves past.

Energy and ambition will be necessary to keep us in the front rank. "We must remember that it takes a live fish to swim up stream, -any dead one can float down."

\title{
LOCAL ANESTHESIA.
}

\section{INFILTRATION AND CONDUCTIVE METHODS.}

\author{
By Kurt H. Thoma, D. D. S., Boston, Mass.
}

\section{$\mathrm{T}$} HERE are today only very few cases of dental surgery, where local anesthesia is not used to greater advantage, with more safety, promising greater success of the operation, and less discomfort to the patient than general anesthesia. The only cases where it cannot be used, are severe cases of ankylosis of the mandible.

To put patients to the strain and danger of ether, chloroform, or similar general anesthesia, with their psychic, and physic prae and after effects, for difficult cases of exodontia, thus confining a patient for days to the hospital, or the bed, is no more justifiable. Let us compare two operations side by side, one under general anesthesia, the other under lo- cal anesthesia; with the former we have general relaxation of the patient, the field of operation continuously obscured by mouth prop,increased venous bleeding and saliva, not to speak of blood and pus, which is swallowed and penetrates into the lungs, and the operation prolonged by endless sponging, which takes up more than half of the time. Under local anesthesia we have a clear view, bleeding almost obliterated, and co-operation of the patient. Between these two it is easy for me to choose, and it also makes it clear why the choice is for the operator and not for the patient.

But local anesthesia does more than replace general anesthesics. It increases the possibility of painless dentistry. With 